\title{
Design of Control System for the De-icing Robot on Transmission Line
}

\author{
Jixin Liu ${ }^{1, a}$, Fengju $\mathrm{Hu}^{2, \mathrm{~b}}$, Shixian Zeng ${ }^{3, \mathrm{c}}$ and Shuangfeng $\mathrm{Han}^{4, \mathrm{~d}}$ \\ $1,2,3,4$ Qing Dao Huang Hai University, P.R.China \\ asdljxin@sina.com, bsdhfju@sina.com, ${ }^{\mathrm{c}}$ 12893317@qq.com, ${ }^{\mathrm{d}} 869313750 @ q q . c o m$
}

\begin{abstract}
A new type of control system for the De-icing Robot on high voltage transmission line is designed in the article. Using the control system, the De-icing Robot can not only get rid of the ice of transmission line effectively, but also can detect the basic information, and comprehend the deicing and line inspection.
\end{abstract}

Keywords: De-icing Robot, Expert control system (EC), Hierarchical control system

\section{Introduction}

With the development of the social economy and the improvement of the peoples' living standard, the safe and stable operation of power grid has become more and more important. However, in recent years, the safety of power grids has become threatened by the natural disasters such as hail in fig. 1 . For the most part, this has become a serious problem the northern areas of China, causing inconvenience to the normal operation of the power grid and everyone's everyday life.

A transmission line deicing is especially important to reduce the number of accidents, whereas the traditional manual deicing spends a lot of resources and inefficiency ${ }^{[1,2,3]}$. Thus, it has immediate practical significance and important value in development of transmission line De-icing Robot instead of traditional manual deicing.

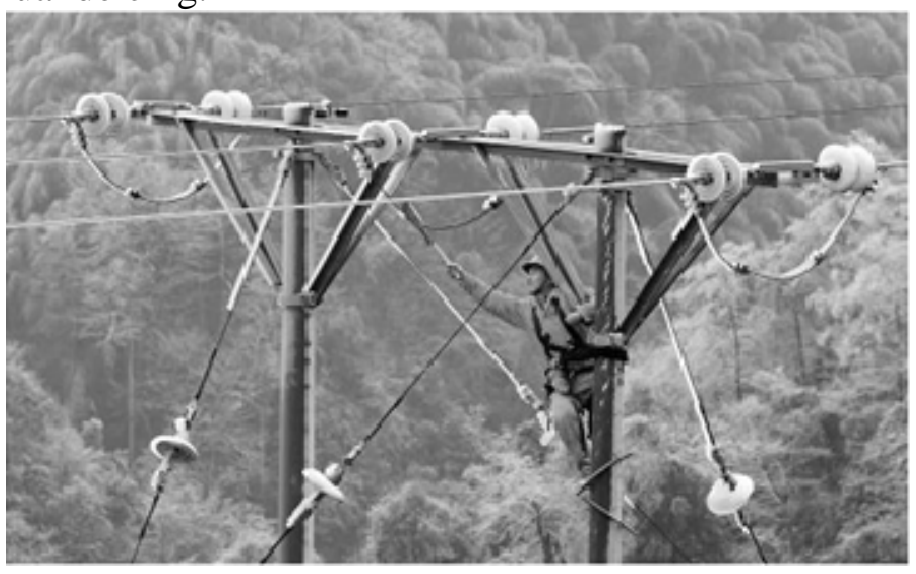

Fig. 1 The icing condition of transmission line

\section{General introduce}

De-icing Robot, such as fig. 2, which we have researched, mainly includes mobile mechanism, sensing system and control systems. Its main mechanical structure includes walking mechanism, deicing mechanism, brake mechanism, telescopic mechanism, etc. The middle arm is equipped with a walking mechanism (including two walking wheels) and a brake device. All three arms are installed a telescopic mechanism, which can achieve arm scaling throughout walking. the rotating joint is installed in the vertical and horizontal direction for rotating arms. By controlling the control cabinets, forward or backward, barycenter adjusting mechanism can adjust the robot's barycenter.

Since it is a mobile robot, De-icing Robot is in suspended state because of its working environment (overhead HV transmission line) and needs to run smoothly on the high voltage 
transmission lines, which needs higher requirements for its control system. Path Settings and modeling approach of the ground mobile robot is no longer suitable for modern De-icing $\operatorname{Robot}^{[4,5]}$.

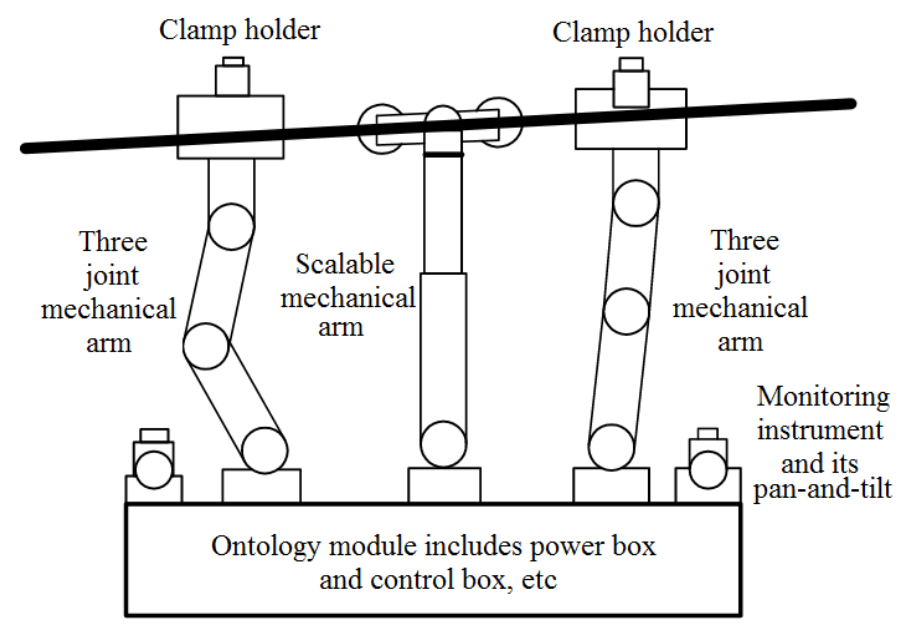

Fig. 2 Human institutions figure of De-icing Robot

This device, depending on De-icing Robot control theory and the modern intelligent control theory, design a new type of control system for the De-icing Robot. With it controlled, the De-icing Robot can not only get rid of the ice of transmission line effectively, but also can detect the basic information, and recognize the deicing and line inspection.

\section{Working principle}

De-icing Robot, using three mechanical arms, can satisfy its working stability, overcome various circuitry obstacles, and understand deicing or walking on the transmission line. The fig. 3 shows its working principle.

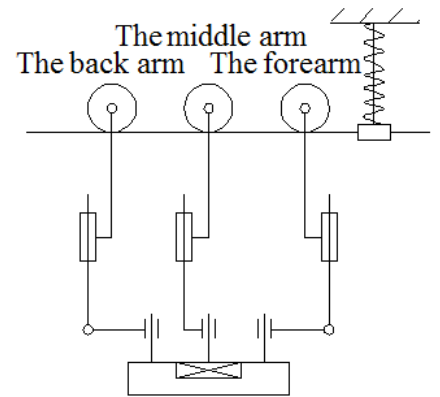

(a) The original location

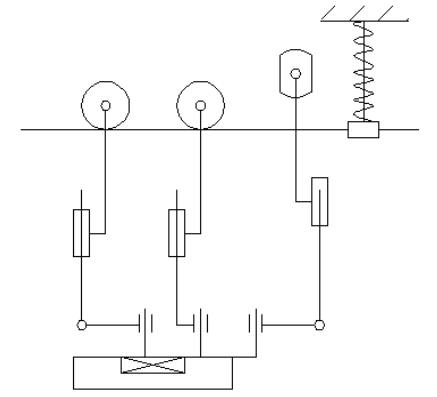

(b) Raised the forearm,

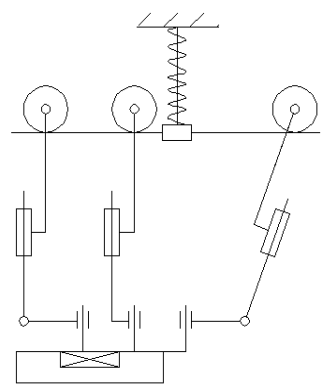

(c) The forearm obstacle online side swing, and move backward the control box

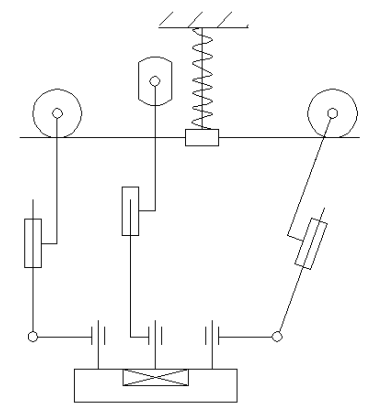

(d) Raised and side swing The middle arm

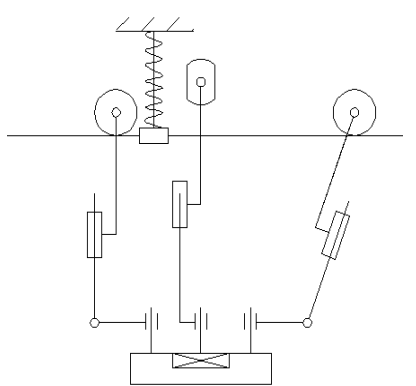

(e) Forward the whole machine

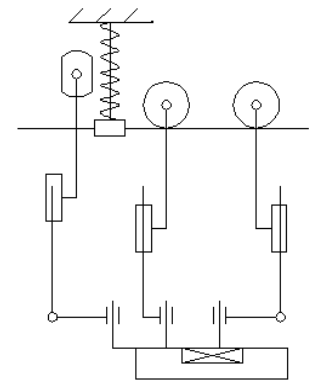

(f) Raised and side swing the back arm, forward and return control box 


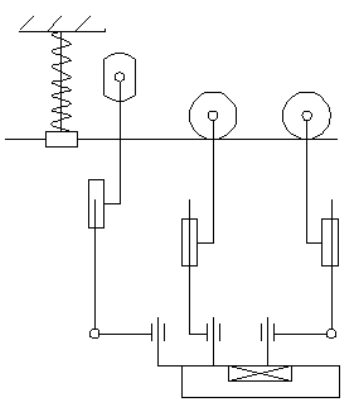

(g) Forward the whole machine

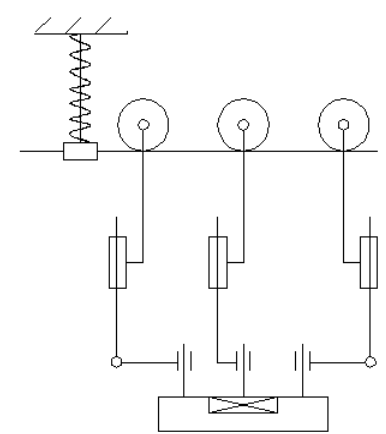

(h) Drop and go online the back arm, return the control box

Fig. 3 Working process of the De-icing Robot

Raise and lower the arm, hang line and off-line the hand, and rotate the shoulder joint, all of which are basic action of the robot. Meanwhile, coordinating these basic actions can comprehend surmounting obstacles ${ }^{[6]}$. The key to autonomous obstacle negotiation is judging the location of obstacle and the robot posture, which is on the information fusion of the sensors and synthesizing them.

\section{Design the control system}

The expert control system can better meet the requirements about control system of the De-icing Robot and improve its intelligent level. Expert system has many characteristics, such as high real time, better anti-jamming, high reliability and operational integrity, etc ${ }^{[7]}$. The system will use expert systems based on regulation, which is according to the latest development of expert control system. The expert system, based on regulation is a new information system that uses knowledge base to deal with the facts of working memory. The complete structure of expert system based on regulation is shown in fig. 4 .

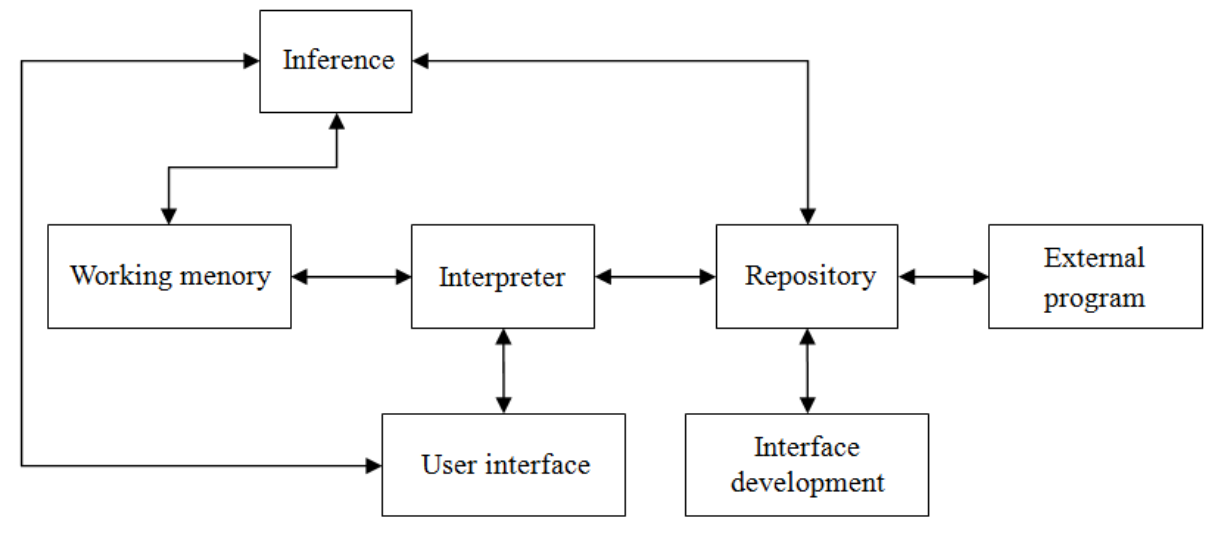

Fig. 4 The expert system model

Among the expert system model, repository, inference engine and working memory are the core.

(1) Repository

In this expert system, repository, also known as rule base, is a set of rule models that sets up peoples' long-term memory. Repository generally adopts the IF... THEN to display rules.

(2) Working memory

Working memory, also known as fact memory, is a set of rule models that set up peoples' short-term memory. This is to store facts and other memories that are derived by rules.

(3) Inference engine

The inference engine builds up an expert reasoning model to infer the new information by combining,the matter-of-fact stored in working memory with instructions. Stored in repository. Inference engine compares the precondition of facts with rules and then can obtain activated rules. 
Through that, the inference engine puts the conclusion into the working memory, and processes them. During the working process, robotic control systems display the image, thus guides the robot to walk and avoid obstruction and so on. The key to designing robotic control systems consists of the controller making the right decisions to complete complex tasks.

In distributed expert system, independent systems are distributed on the various nodes of the computer network and work together to stay coordinated. Control systems can make the right response in time and meet the requirement of a control system find a different word for the De-icing Robot by simulating the procedure of humans being expert analysis, solving problem and decisions and completing complex tasks.

The deicing structure is shown in fig. 5.

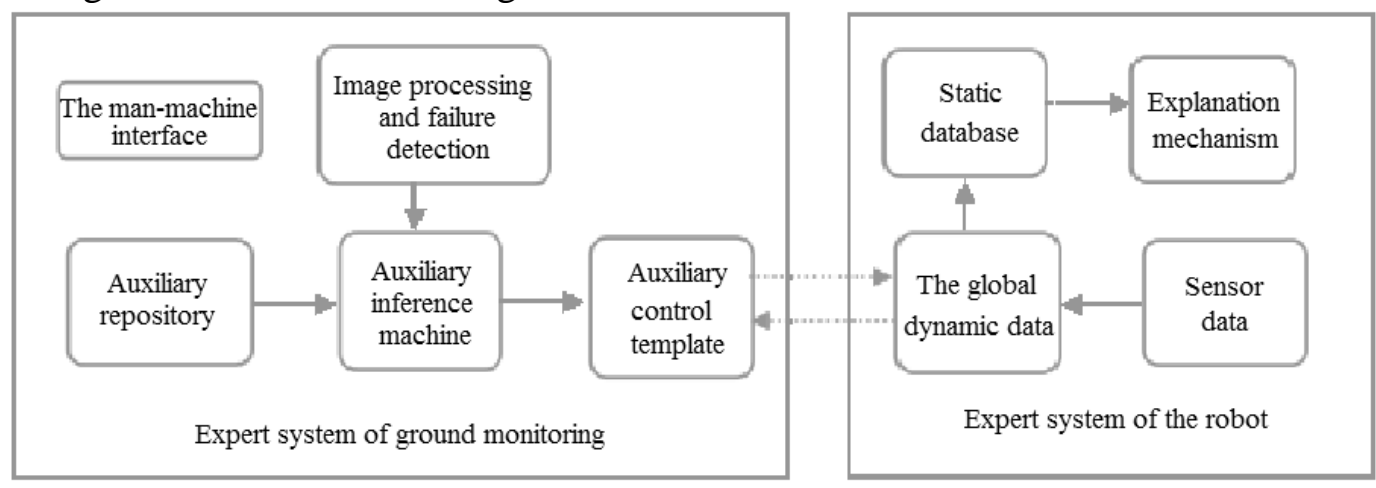

Fig. 5 The expert system structure of the De-icing Robot

There are two-leveled expert control systems (ECS): the expert system of ground monitoring and the robot.

The De-icing Robot control system can understand management and decision-making through its coordination.

The ground expert system is used for disturbance judgement and sending remote commands, processing images and comparing obstacle data. Ground expert system can infer the control command to the organizational. Meanwhile, through the man-machine, interface will receive the command, ground expert system will send it to the robot. Thus, ground crew intuitive judges the line according to the image and follows the remote operation mode by sending corresponding operation command.

To understand the combination of the control system and remote monitoring system, De-icing Robot uses the structure design of hierarchical control system as showed in fig. 6 .

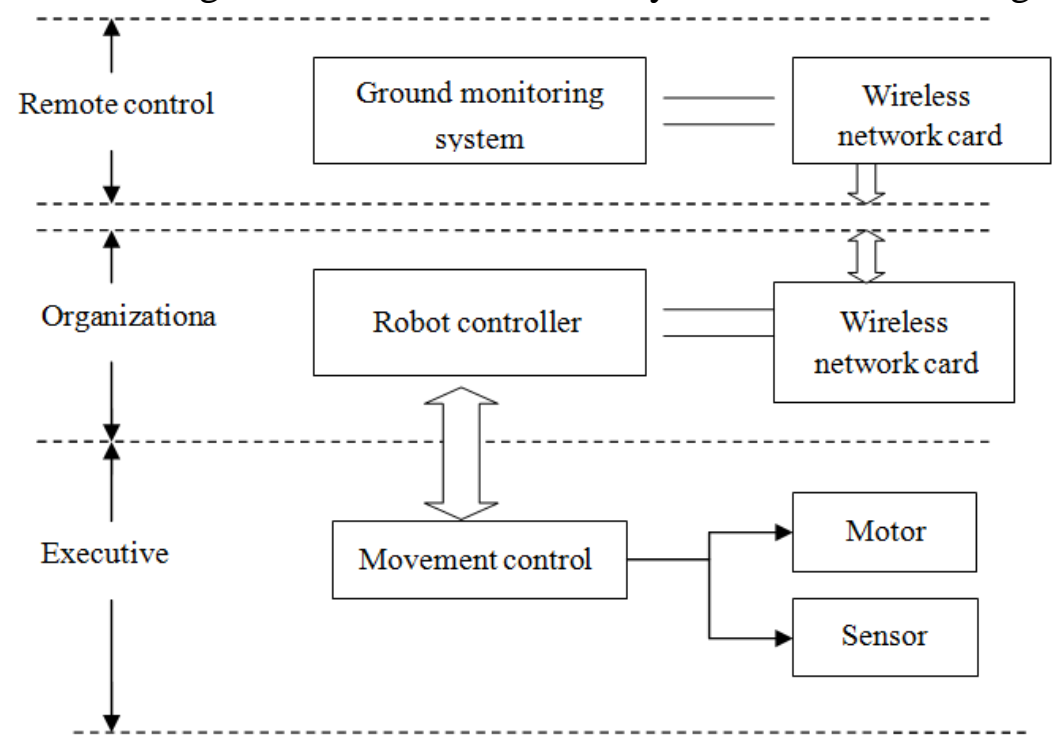

Fig. 6 The control system structure of the De-icing Robot

Remote control, organizational and executive 
The remote control is responsible for the coordination of levels and assisting the organization level. It can mark the image sent by the organization level, and display the image on the ground monitor screen. It also can control De-icing Robot motion and diagnose failure by the pattern of remote control.

The organization is there another word to use, which is the the robot body, is the core of control system. Its responsible is collecting and processing the sensor information and image. This combines sensor information and its own repository, which plans the De-icing Robot movement, which sends the action sequence to the lower computer. Furthermore, it can communicate the remote control level with wireless cards and accept its control information.

The executive is the lowest level in intelligent control system, but has high accuracy. This mainly includes various motor controlled and motion controlled cards. It can receive action sequences from organization, gather operating process and send the data information to the organization.

\section{Conclusion}

The main contribution of this dissertation is summarized as follows:

Design a new mechanical arm of the De-icing Robot on transmission lines. The experiment shows that this structure can not only deice effectively, but also have stronger obstacle-climbing capability and a certain inspection function. This new mechanical arm achieves the function of multipurpose.

Design a new control system of De-icing Robot, combined with the hierarchical control structure and the principle of expert system. Rule-based expert control system and hierarchically control system structure of specific realizing steps are put forward.

Provide a new method for deicing, inspection and maintenance of the De-icing Robot on transmission line.

\section{Acknowledgement}

This work was financially supported by Foundation for 2015 year of University Youth Key Teacher by the Ministry of Education of Shandong province.

\section{References:}

[1] En Li, Zize Liang and Min TAN. Rule Base Based Motion-planning Method for Inspection Robot to Cross Obstacles Autonomously [J]. Robot, 2005, 27 (5).

[2] Xinglong Zhu,Hongguang Wang,Lijin Fang,Mingyang Zhao and Jiping Zhou.Analysis of Drive Dynamic Performance and Position-pose of Autonomous Robot for Transmission Line Inspection [J].Journal of Mechanical Engineering, 2006, 42(12).

[3] Zhenyu Li. Research on Control System of 220kv High-voltage Transmission Inspection Robot [D].Hubei Wuhan: Wuhan University, 2005.

[4] Dong Tang,Lijin Fang and Hongzhi Wang.Inspection of the Robot Control System of Power Transmission Line Based on Distributed Expert System [J].Robot, 2004(3).

[5] Weizan Li, Jidai Wang, Aiqin Sun and Qing Li. Design and the Study of the Control System of a Power Transmission Line Inspection Robot [J].Manufacturing Automation, 2008(11).

[6] Wei Su, Jidai Wang, Aiqin Sun and Dongyue Zhang .Expert Control System of Inspection Robot for High Voltage Transmission Line [J].Computer Engineering, 2012(15).

[7] Dong Tang.Research on Inspection Robot Control System of Power Transmission Line [D].Shenyang Institute of Automation, the Chinese Academy of Sciences, 2004. 